\title{
On the Resilience of Long Cycles in Random Graphs
}

\author{
Domingos Dellamonica Jr* \\ Department of Mathematics and Computer Science, Emory University \\ 400 Dowman Dr., Atlanta, GA, 30322, USA \\ ddellam@mathcs. emory. edu \\ Yoshiharu Kohayakawa ${ }^{\dagger}$ \\ Instituto de Matemática e Estatística, Universidade de São Paulo \\ Rua do Matão 1010, 05508-090 São Paulo, Brazil \\ yoshi@ime.usp.br \\ Martin Marciniszyn Angelika Steger \\ Institute of Theoretical Computer Science \\ ETH Zürich, 8092 Zürich, Switzerland \\ \{mmarcinilsteger\}@inf .ethz.ch
}

Submitted: Jun 13, 2007; Accepted: Feb 5, 2008; Published: Feb 11, 2008

Mathematics Subject Classification: 05C35, 05C38, 05C80

\begin{abstract}
In this paper we determine the local and global resilience of random graphs $G_{n, p}\left(p \gg n^{-1}\right)$ with respect to the property of containing a cycle of length at least $(1-\alpha) n$. Roughly speaking, given $\alpha>0$, we determine the smallest $r_{g}(G, \alpha)$ with the property that almost surely every subgraph of $G=G_{n, p}$ having more than $r_{g}(G, \alpha)|E(G)|$ edges contains a cycle of length at least $(1-\alpha) n$ (global resilience). We also obtain, for $\alpha<1 / 2$, the smallest $r_{l}(G, \alpha)$ such that any $H \subseteq G$ having $\operatorname{deg}_{H}(v)$ larger than $r_{l}(G, \alpha) \operatorname{deg}_{G}(v)$ for all $v \in V(G)$ contains a cycle of length at least $(1-\alpha) n$ (local resilience). The results above are in fact proved in the more general setting of pseudorandom graphs.
\end{abstract}

\footnotetext{
*Supported by a CAPES-Fulbright scholarship.

${ }^{\dagger}$ Partially supported by FAPESP and CNPq through a Temático-ProNEx project (Proc. FAPESP 2003/09925-5) and by CNPq (Proc. 306334/2004-6 and 479882/2004-5).
} 


\section{Introduction}

Problems in extremal graph theory $[1,2]$ usually come in the following form: let $\mathcal{P}$ be a graph property and $\mu$ a graph parameter; determine the least $m$ with the property that any graph $G$ with $\mu(G)>m$ has $\mathcal{P}$ and describe the so called extremal graphs, that is, those $G$ with $\mu(G)=m$ without $\mathcal{P}$. For instance, in the case of Turán's theorem, $\mathcal{P}$ is the property of containing a clique $K_{t}$ for some given $t$, and $\mu(G)$ is the number of edges $e(G)$ in $G$. As is well known, Turán's classical result determines the exact value of $m=m(n)$ in terms of $n=|V(G)|$ and describes all the extremal graphs.

In this paper, we consider properties $\mathcal{P}$ that are increasing and non-trivial, in the sense that graphs with no edges do not have $\mathcal{P}$. We are interested in the resilience of certain graph families with respect to such properties. In simple terms, the resilience is a measure of how strongly a graph $G$ possesses property $\mathcal{P}$. Sudakov and Vu [24] define this notion as follows.

Definition 1 (Global resilience). Let $\mathcal{P}$ be an increasing monotone property. The global resilience of a graph $G$ with respect to $\mathcal{P}$ is the minimum number $r_{g}=r_{g}(G, \mathcal{P})$ such that one can destroy $\mathcal{P}$ by deleting at most $r_{g} \cdot e(G)$ edges from $G$.

In some cases, the following variant of resilience makes more sense.

Definition 2 (Local resilience). Let $\mathcal{P}$ be an increasing monotone property. The local resilience of a graph $G$ with respect to $\mathcal{P}$ is the minimum number $r_{l}=r_{l}(G, \mathcal{P})$ such that one can destroy $\mathcal{P}$ by deleting at most $r_{l} \cdot \operatorname{deg}(v)$ edges at each vertex $v$ from $G$.

Determining the resilience of a graph $G$ w.r.t. $\mathcal{P}$ can be viewed as follows. Suppose an adversary is allowed to remove up to a certain number $R$ of edges from $G$ globally, with the aim of destroying $\mathcal{P}$. If $R<r_{g}(G, \mathcal{P}) \cdot e(G)$ the graph left by the adversary will necessarily have $\mathcal{P}$. If $R \geq r_{g}(G, \mathcal{P}) \cdot e(G)$, then the adversary has a strategy to obtain a graph that does not have $\mathcal{P}$. The notion of local resilience corresponds to a variant in which the adversary has to obey a local rule: for any vertex $v \in V(G)$, no more that $r_{l}(G, \mathcal{P}) \cdot \operatorname{deg}(v)$ edges incident to $v$ may be removed.

Our general problem here is to study $r_{g}(G, \mathcal{P})$ and $r_{l}(G, \mathcal{P})$ in the case in which $G$ is a (pseudo)random graph and $\mathcal{P}$ concerns the containment of a large cycle. We shall consider the classical $G_{n, p}$ model of binomial random graphs, that is, $G_{n, p}$ consists of $n$ labeled vertices, and the edges are independently present with probability $p=$ $p(n)$. We mention that several authors have investigated $r_{g}(G, \mathcal{P})$ and $r_{l}(G, \mathcal{P})$ in random or pseudorandom graphs for various properties $\mathcal{P}$. For instance, the global 
resilience of $G_{n, p}$ with respect to the Turán property of containing a clique $K_{t}$ of a given order, or, more generally, the property of containing a given graph $H$ of fixed order was studied in [18], [20], and [25]; for the case in which $H$ is a cycle, see [10], [13], and [14] (for further related results, see [16], [17], and [21]). More recently, Sudakov and $\mathrm{Vu}[24]$ determined the local resilience of $G_{n, p}$ with respect to several properties, namely having a perfect matching, being Hamiltonian, being non-symmetric, and being $k$-colorable for a given function $k=k(n)$. Similar results concerning Hamiltonicity were obtained by Frieze and Krivelevich [8].

In this paper, we study the resilience of random graphs w.r.t. having a cycle of length proportional to the number of vertices $n$. The circumference $\operatorname{circ}(G)$ of a graph $G$ is the length of a longest cycle in $G$. A classical theorem of Erdös and Gallai [7] (see also, e.g., Bollobás [1, 2, Chapter 3, Sect. 4]) gives a sufficient condition on the number of edges in any graph $G$ on $n$ vertices for the circumference of $G$ to be greater than $\ell, 3 \leq \ell \leq n$. Woodall [26] proved a strengthening of this result for the case in which $n-1$ is not divisible by $\ell-2$.

Theorem 3 (Woodall [26]). Let integers $3 \leq \ell \leq n$ be given. Every graph $G$ on $n$ vertices with

$$
e(G) \geq\left\lfloor\frac{n-1}{\ell-2}\right\rfloor\left(\begin{array}{c}
\ell-1 \\
2
\end{array}\right)+\left(\begin{array}{c}
r+1 \\
2
\end{array}\right)+1
$$

$r=(n-1) \bmod (\ell-2)$, satisfies $\operatorname{circ}(G) \geq \ell$.

The reader is referred to the book of Bollobás [1,2] as well as to the surveys of Bondy [3] and Simonovits [23] for related problems and historical information. Theorem 3 was reproved by Caccetta and Vijayan in [4]. The bound in Theorem 3 is best possible for all integers $n$. Consider, for instance, the graph $G$ on $n$ vertices that is a collection of $\lfloor(n-1) /(\ell-2)\rfloor$ cliques of size $\ell-2$ plus one additional clique of size $r$ such that all members of this collection are completely connected to another vertex $v$. Clearly, this construction does not allow for a cycle in $G$ of length greater than $\ell-1$.

With respect to cycles of length proportional to $n$, Theorem 3 yields the following result; see Section 3.1 for a proof.

Corollary 4. Let $\alpha>0$ be given. Then, for every $\beta>0$, there exists $n_{0}$ such that every graph $G$ on $n \geq n_{0}$ vertices with

$$
e(G) \geq\{1-(1-\mathrm{w}(\alpha))(\alpha+\mathrm{w}(\alpha))+\beta\}\left(\begin{array}{l}
n \\
2
\end{array}\right)
$$

where

$$
\mathrm{w}(\alpha)=1-(1-\alpha)\left\lfloor(1-\alpha)^{-1}\right\rfloor
$$


satisfies $\operatorname{circ}(G) \geq(1-\alpha) n$.

We state our results in terms of pseudorandom graphs. Using the fact that truly random graphs are asymptotically almost surely, i.e., with probability tending to 1 as $n$ tends to infinity, pseudorandom, enables us to formulate and prove all statements without involving probability. Below, we write $e_{G}(U, W)$ for the number of edges with one endpoint in $U$ and the other endpoint in $W$.

Definition 5. A graph $G$ on $n$ vertices is $(p, A)$-uniform if, for $d=p n$, we have

$$
\left|e_{G}(U, W)-p\right| U|| W|| \leq A \sqrt{d|U||W|}
$$

for all disjoint sets $U, W \subseteq V(G)$ such that $1 \leq|U| \leq|W| \leq d|U|$. We call $G$ $(p, A)$-upper-uniform if the bound for the upper deviation in (1) holds, i.e.,

$$
e_{G}(U, W) \leq p|U||W|+A \sqrt{d|U||W|} .
$$

In $(p, A)$-uniform graphs $G$, the number of edges induced by a set $U$ of vertices is under tight control. It can be observed by a double counting argument that, for any $U \subset V(G)$, we have

$$
\left|e(G[U])-p\left(\begin{array}{c}
|U| \\
2
\end{array}\right)\right| \leq A \sqrt{d}|U| .
$$

See [6] for a proof. As long as $A$ is a sufficiently large constant, $(p, A)$-uniform graphs are abundant. The following lemma is proved in [12].

Lemma 6. For every $0<p=p(n) \leq 1$ the random graph $G_{n, p}$ is $\left(p, \mathrm{e}^{2} \sqrt{6}\right)$-uniform with probability $1-o(1)$.

With these definitions at hand we can now state our first main result, which can be viewed as the counterpart of the theorems of Erdős and Gallai [7] and Woodall [26] for $(p, A)$-uniform graphs.

Theorem 7. Suppose $A>0$ is fixed, and we have $p=p(n) \gg n^{-1}$. Then, for all $\alpha>0$, all $(p, A)$-uniform graphs $G$ on $n$ vertices satisfy the following property: The global resilience of $G$ with respect to having circumference greater than $(1-\alpha) n$ is

$$
(1-\mathrm{w}(\alpha))(\alpha+\mathrm{w}(\alpha))+o(1)
$$

where

$$
\mathrm{w}(\alpha)=1-(1-\alpha)\left\lfloor(1-\alpha)^{-1}\right\rfloor
$$


A classical result of Dirac [5] states that any graph on $n \geq 3$ vertices with minimum degree at least $n / 2$ contains a Hamiltonian cycle. By combining ideas from the proof of Theorem 7 with this classical theorem from graph theory, we obtain our other main result, which states that by removing up to a little less than one half of all the edges incident to any vertex in a relatively sparse $(p, A)$-uniform graph $G$, an adversary cannot destroy all long cycles in $G$.

Theorem 8. Suppose $A>0$ is fixed, and we have $p=p(n) \gg n^{-1}$. Then, for all $0<$ $\alpha<1 / 2$, all $(p, A)$-uniform graphs $G$ on $n$ vertices satisfy the following property: The local resilience of $G$ with respect to having circumference greater than $(1-\alpha) n$ is

$$
\frac{1}{2}+o(1)
$$

In Section 2 we introduce a variant of Szemerédi's Regularity Lemma for $(p, A)$ upper-uniform graphs and state our main technical lemma that shows how to embed long paths into regular pairs. Theorems 7 and 8 are proved in Section 3.

\section{Regularity and long paths}

In Section 2.1 we present a variant of Szemerédi's Regularity Lemma for sparse graphs. We employ a version of the lemma tailored to $(p, A)$-uniform graphs. Section 2.2 comprises the proof of our main technical lemma, Lemma 10. This lemma states that dense, regular pairs permit an almost complete covering by a long path.

\subsection{Szemerédi's Regularity Lemma for sparse graphs}

Let a graph $G=(V, E)$ and a real number $0<p \leq 1$ be given. We define the $p$ density of a pair of non-empty, disjoint sets $U, W \subseteq V$ in $G$ by

$$
d_{G, p}(U, W)=\frac{e_{G}(U, W)}{p|U||W|} .
$$

For any $0<\varepsilon \leq 1$, the pair $(U, W)$ is said to be $(\varepsilon, G, p)$-regular, or $(\varepsilon, p)$-regular or even just p-regular for short, if, for all $U^{\prime} \subseteq U$ with $\left|U^{\prime}\right| \geq \varepsilon|U|$ and all $W^{\prime} \subseteq W$ with $\left|W^{\prime}\right| \geq \varepsilon|W|$, we have

$$
\left|d_{G, p}(U, W)-d_{G, p}\left(U^{\prime}, W^{\prime}\right)\right| \leq \varepsilon .
$$

We say that a partition $\Pi=\left(V_{0}, V_{1}, \ldots, V_{k}\right)$ of $V$ is $(\varepsilon, G, p)$-regular if $\left|V_{0}\right| \leq \varepsilon|V|$ and $\left|V_{i}\right|=\left|V_{j}\right|$ for all $i, j \in\{1,2, \ldots, k\}$, and, furthermore, at least $(1-\varepsilon)\left(\begin{array}{l}k \\ 2\end{array}\right)$ pairs $\left(V_{i}, V_{j}\right)$ with $1 \leq i<j \leq k$ are $(\varepsilon, G, p)$-regular. 
Recall the notion of $(p, A)$-upper-uniform graphs as stated in Definition 5 . We combine this with the following variant of Szemerédi's Regularity Lemma for sparse graphs (see, e.g., $[9,15,19])$.

Lemma 9. For all real numbers $\varepsilon>0$ and $A \geq 1$ and all integers $k_{0}$, there exist constants $n_{0}=n_{0}\left(\varepsilon, A, k_{0}\right)>0, d_{0}=d_{0}\left(\varepsilon, A, k_{0}\right)>0$, and $K_{0}=K_{0}\left(\varepsilon, A, k_{0}\right) \geq k_{0}$ such that the following holds. For every $(p, A)$-upper-uniform graph $G$ on $n \geq n_{0}$ vertices with $d=p n \geq d_{0}$, there exists a partition $\Pi=\left(V_{0}, \ldots, V_{k}\right)$ of $V$ with $k_{0} \leq$ $k \leq K_{0}$ that is $(\varepsilon, G, p)$-regular.

We remark that Lemma 9 holds under weaker hypotheses on the graphs $G$, but for the purpose of this work the above will do.

\subsection{Long paths in regular pairs}

This section is devoted to the proof of the following result, which guarantees long paths in $(\varepsilon, p)$-regular pairs provided that those are $(A, p)$-upper-uniform for a given constant $A$. Lemma 10 is the main technical ingredient in the proof of our theorems.

Lemma 10. For all $0<\varrho, \mu \leq 1 / 2$, there exists $\varepsilon=\varepsilon(\varrho, \mu)>0$ and, for all $0<$ $\nu \leq 1$ and $A>0$, there exists $d_{0}=d_{0}(\varrho, \mu, \nu, A)$ such that the following holds. Let $G$ be a $(p, A)$-upper-uniform graph on $n$ vertices and $d=p n \geq d_{0}$. Suppose that $V_{1}, V_{2} \subseteq V(G)$ satisfy

(i) $V_{1} \cap V_{2}=\emptyset$;

(ii) $\left|V_{1}\right|=\left|V_{2}\right|=m \geq \nu n$;

(iii) the induced bipartite graph $G\left[V_{1}, V_{2}\right]$ is $(\varepsilon, p)$-regular with density $d_{1,2}:=d_{G, p}\left(V_{1}, V_{2}\right) \geq \varrho$.

Then there exist sets $X \subseteq V_{1}$ and $Y \subseteq V_{2}$ of size at least $\varepsilon m$ such that any $x \in X$ and any $y \in Y$ are endpoints of a path on at least $2(1-2 \mu) m$ vertices in $G\left[V_{1}, V_{2}\right]$.

We shall prove Lemma 10 in the remainder of this section. Our approach is similar to the proof of Lemma 2.7 in [6]. We say that a bipartite graph $B=(U \dot{\cup} W, E)$ is $(b, f)$-expanding if for every set $X \subseteq U$ and every set $Y \subseteq W,|X|,|Y| \leq b$, we have

$$
|\Gamma(X)| \geq f|X| \text { and }|\Gamma(Y)| \geq f|Y|
$$

Here, as usual, $\Gamma(Z)$ denotes the neighborhood of a vertex-set $Z$, that is, the set of all vertices adjacent to some $z \in Z$.

We make use of the following result, which is a variant of a well known lemma due to Pósa [22] (for a proof, see [11]). 
Lemma 11. Let $b \geq 1$ be an integer. If the bipartite graph $B$ is $(b, 2)$-expanding, then $B$ contains a path on $4 b$ vertices.

Proof of Lemma 10. Let

$$
\varepsilon=\frac{\mu^{2} \varrho}{8}
$$

Moreover, let

$$
\delta=\varepsilon\left(\mu^{-1}+\varrho^{-1}\right)
$$

and choose $d_{0}$ such that

$$
d_{0}\left(\frac{\delta \varrho \mu \nu}{A}\right)^{2} \geq 2
$$

Claim 12. For all $V_{1}^{\prime} \subseteq V_{1}$ and $V_{2}^{\prime} \subseteq V_{2}$ of size at least $\mu m$, there exist $U_{1} \subseteq V_{1}^{\prime}$ and $U_{2} \subseteq V_{2}^{\prime}$ of size at least $(\mu-\varepsilon) m$ such that for all $u_{1} \in U_{1}$ and all $u_{2} \in U_{2}$, we have

$$
\left|\Gamma\left(u_{1}\right) \cap U_{2}\right| \geq(1-\delta) d_{1,2} p \mu m \quad \text { and } \quad\left|\Gamma\left(u_{2}\right) \cap U_{1}\right| \geq(1-\delta) d_{1,2} p \mu m
$$

respectively.

Proof. We inductively define a sequence

$$
\mathbf{B}(t)=\left(V_{1}(t), V_{2}(t)\right) \quad(t=0,1,2, \ldots)
$$

as follows. Start with $\mathbf{B}(0)=\left(V_{1}^{\prime}, V_{2}^{\prime}\right)$. Suppose now that $t \geq 0$ and that we have computed $\mathbf{B}(t)$. If $(6)$ is satisfied for $U_{1}=V_{1}(t)$ and $U_{2}=V_{2}(t)$, we are done. Otherwise, take

$$
V_{i}(t+1)=V_{i}(t) \backslash\{x\}
$$

for some $x \in V_{i}(t)$ and $i$ such that $\left|\Gamma(x) \cap V_{j}(t)\right|<(1-\delta) d_{1,2} p \mu m$ for $j \neq i$ with $1 \leq$ $i, j \leq 2$; moreover, take $V_{j}(t+1)=V_{j}(t)$.

Let us suppose for a contradiction that, at some moment $T$, we have, without loss of generality, $\left|V_{1}(T)\right|<(\mu-\varepsilon) m$ and $\left|V_{2}(T)\right| \geq(\mu-\varepsilon) m$.

Let $X:=V_{1}^{\prime} \backslash V_{1}(T)$. Clearly, we have $|X| \geq \varepsilon m$ and, for all $x \in X$, we have $\left|\Gamma(x) \cap V_{2}(T)\right|<(1-\delta) d_{1,2} p \mu m$. It follows that

$$
e\left(X, V_{2}(T)\right)<(1-\delta) d_{1,2} p \mu m|X|,
$$

which implies that the $p$-density of the pair $\left(X, V_{2}(T)\right)$ is $d_{G, p}\left(X, V_{2}(T)\right)<(1-\delta) d_{1,2} \frac{\mu m}{\left|V_{2}(T)\right|} \leq\left(1-\frac{\delta \mu-\varepsilon}{\mu-\varepsilon}\right) d_{1,2}<\left(1-\frac{\varepsilon}{\varrho}\right) d_{1,2} \leq d_{1,2}-\varepsilon$.

This, however, contradicts the regularity of the pair $\left(V_{1}, V_{2}\right)$. 
Claim 13. The bipartite graph induced by $U_{1}$ and $U_{2}$ given in Claim 12 is $((1-$ $\left.2 \delta) d_{1,2} \mu m / f, f\right)$-expanding for any $0<f \leq(\delta \varrho \nu \mu / A)^{2} d$.

Proof. Let $X \subseteq U_{i}, 1 \leq i \leq 2$, be such that $|X| \leq(1-2 \delta) d_{1,2} \mu m / f$. Let $Y=$ $\Gamma(X) \cap U_{j}$ with $j \neq i$ and suppose that $|Y|<f|X|$.

By the upper-uniformity condition on $G$, we have

$$
e(X, Y) \leq p|X||Y|+A \sqrt{d|X||Y|}<p|X|(1-2 \delta) d_{1,2} \mu m+A \sqrt{d|X||Y|},
$$

and, from (6), we deduce that

$$
e(X, Y)=e\left(X, U_{j}\right) \geq(1-\delta) d_{1,2} p \mu m|X| .
$$

Combining (7) and (8), we have that $\left(\delta d_{1,2} p \mu m|X|\right)^{2}<A^{2} d|X||Y|$. Therefore,

$$
|Y|>\frac{\left(\delta d_{1,2} p \mu m|X|\right)^{2}}{A^{2} d|X|} \geq\left(\frac{\delta \varrho \nu \mu}{A}\right)^{2} d|X| \geq f|X|,
$$

a contradiction.

We continue the proof of Lemma 10 by iterative applications of Claims 12 and 13. Let

$$
b=\left\lfloor\frac{1}{2}(1-2 \delta) \varrho \mu m\right\rfloor .
$$

Construct a sequence of disjoint paths $P(t), t=1,2, \ldots$, on the vertices in $V_{1} \cup V_{2}$ each of length $4 b$ as follows. Suppose $P(1), \ldots, P(t-1)$ have already been obtained. We build $P(t)$ in the following way. Let

$$
V_{1}^{\prime}=V_{1} \backslash \bigcup_{j=1}^{t-1} V(P(j)) \text { and } \quad V_{2}^{\prime}=V_{2} \backslash \bigcup_{j=1}^{t-1} V(P(j))
$$

Observe that $\left|V_{1}^{\prime}\right|=\left|V_{2}^{\prime}\right|$ since all paths have even length. If $\left|V_{1}^{\prime}\right| \geq \mu m$, then we can apply Claim 12 in order to obtain sets $U_{1} \subseteq V_{1}^{\prime}$ and $U_{2} \subseteq V_{2}^{\prime}$ of size at least $(\mu-\varepsilon) m$. It follows from Claim 13 and the choice of $d$ (see $(5))$ that $\left(U_{1}, U_{2}\right)$ is $(b, 2)$-expanding. Therefore, we obtain a path $P(t)$ of length $4 b$ on the vertices in $U_{1} \cup U_{2}$ by Lemma 11 . We stop constructing new paths as soon as $\left|V_{1}^{\prime}\right|<\mu m$.

Suppose this procedure stopped after $T$ iterations. We concatenate the paths $P(t), 1 \leq t \leq T$, into a single path $P_{0}$ in the following way. Let head $(P(t))$ denote the first $\lceil\varepsilon m\rceil$ vertices of $P(t)$ in $V_{1}$ and analogously tail $(P(t))$ the last $\lceil\varepsilon m\rceil$ vertices 
of $P(t)$ in $V_{2}(1 \leq t \leq T)$. Since $\left(V_{1}, V_{2}\right)$ is $(\varepsilon, p)$-regular with density $d_{1,2}$, we have, for all $2 \leq t \leq T$,

$$
e(\operatorname{tail}(P(t-1)), \operatorname{head}(P(t))) \geq\left(d_{1,2}-\varepsilon\right) p \varepsilon^{2} m^{2} \geq 1
$$

for $m$ sufficiently large. Hence, connecting $P(t-1)$ and $P(t)$ by an arbitrary edge between tail $(P(t-1))$ and head $(P(t))$ yields $P_{0}$ of length at least

$$
2(1-\mu) m-4(T-1)(\lceil\varepsilon m\rceil-1) \geq 2(1-\mu) m-4(T-1)\lfloor\varepsilon m\rfloor
$$

vertices long. Let

$$
X=\operatorname{head}\left(P_{0}\right) \quad \text { and } \quad Y=\operatorname{tail}\left(P_{0}\right)
$$

Then any $x \in X$ and any $y \in Y$ are endpoints of a path of length at least

$$
2(1-\mu) m-4 T\lfloor\varepsilon m\rfloor \geq 2\left(1-\mu-\frac{8 \varepsilon}{\varrho \mu}\right) m \geq 2(1-2 \mu) m
$$

since

$$
T \leq \frac{m}{2 b} \leq \frac{m}{(1-2 \delta) \varrho \mu m-2} \leq \frac{3}{2 \varrho \mu} .
$$

In the last inequality we used that, by the choice of $\varepsilon$ and $\delta$, we have

$$
2 \delta=2 \varepsilon\left(\mu^{-1}+\varrho^{-1}\right) \leq \frac{1}{4} \mu(\varrho+\mu) \leq \frac{1}{4} .
$$

This concludes the proof of Lemma 10.

\section{Proofs of Theorems 7 and 8}

We present the proof of Theorem 7 in Section 3.1 and of Theorem 8 in Section 3.2, respectively. Both heavily depend on the results presented in Section 2.2.

\subsection{Proof of Theorem 7}

Proving Theorem 7 requires to show both an upper and a lower bound on the global resilience $r_{g}$ of $G$ w.r.t. containing long cycles. 


\subsubsection{Proof of the upper bound for $r_{g}$}

For the upper bound, it suffices to provide an appropriate strategy for the adversary to destroy all cycles of length at least $(1-\alpha) n$ in a $(p, A)$-uniform graph $G$. One way of achieving that is to partition the vertex set $V(G)$ into $k$ classes of size $(1-$ $\alpha) n$, where $k:=\lfloor 1 /(1-\alpha)\rfloor$, and one additional class for the remaining vertices

of size $\mathrm{w}(\alpha) n$. Clearly, if one deletes all edges with endpoints in distinct partition classes, only cycles of length at most $(1-\alpha) n$ remain in the graph. Since the number of edges between any pair of classes is bounded, the adversary deletes at most

$$
\begin{gathered}
\left(\left(\begin{array}{l}
k \\
2
\end{array}\right)(1-\alpha)^{2}+k(1-\alpha) \mathrm{w}(\alpha)\right)\left(p n^{2}+A \sqrt{d(1-\alpha)^{2} n^{2}}\right) \\
=(1-\alpha) k((1-\alpha)(k-1)+2 \mathrm{w}(\alpha))(1+o(1)) p\left(\begin{array}{l}
n \\
2
\end{array}\right)
\end{gathered}
$$

and using the identity $(1-\alpha) k=1-\mathrm{w}(\alpha)$

$$
\begin{aligned}
& =(1-\mathrm{w}(\alpha))(\alpha-\mathrm{w}(\alpha)+2 \mathrm{w}(\alpha))(1+o(1)) p\left(\begin{array}{l}
n \\
2
\end{array}\right) \\
& =((1-\mathrm{w}(\alpha))(\alpha+\mathrm{w}(\alpha))+o(1)) e(G)
\end{aligned}
$$

edges from $G$, and the upper bound is proved.

\subsubsection{Proof of the lower bound for $r_{g}$}

We start by proving Corollary 4 .

Proof of Corollary 4. We apply Theorem 3 with $\ell=\lceil(1-\alpha) n\rceil$. Rewrite $r=(n-1)$ $\bmod (\ell-2)$ as follows:

$$
\begin{aligned}
r & =n-1-(\lceil(1-\alpha) n\rceil-2)\left\lfloor\frac{n-1}{\lceil(1-\alpha) n\rceil-2}\right\rfloor \\
& =n-1-\left(1-\alpha+O\left(n^{-1}\right)\right)\left\lfloor\frac{1+O\left(n^{-1}\right)}{1-\alpha+O\left(n^{-1}\right)}\right\rfloor n \\
& =\left(\left(1-(1-\alpha)\left\lfloor(1-\alpha)^{-1}\right\rfloor\right)+O\left(n^{-1}\right)\right) n \\
& =(\mathrm{w}(\alpha)+o(1)) n .
\end{aligned}
$$

Now, suppose that the circumference of graph $G$ is strictly less than $\ell$. Then, by 
Theorem 3, we have

$$
\begin{aligned}
e(G) & <\left\lfloor\frac{n-1}{\ell-2}\right\rfloor\left(\begin{array}{c}
\ell-1 \\
2
\end{array}\right)+\left(\begin{array}{c}
r+1 \\
2
\end{array}\right)+1 \\
& =\frac{(n-1-r)(\ell-1)}{2}+\left(\begin{array}{c}
r+1 \\
2
\end{array}\right)+1 \\
& =(1-\mathrm{w}(\alpha)+o(1))(1-\alpha)\left(\begin{array}{l}
n \\
2
\end{array}\right)+\left(\mathrm{w}(\alpha)^{2}+o(1)\right)\left(\begin{array}{l}
n \\
2
\end{array}\right) \\
& =(1-(1-\mathrm{w}(\alpha))(\alpha+\mathrm{w}(\alpha))+o(1))\left(\begin{array}{l}
n \\
2
\end{array}\right),
\end{aligned}
$$

which contradicts the assumption in Corollary 4.

Observe that $\mathrm{w}(\alpha)=0$ if $1-\alpha=1 / k$ for some integer $k$. In that case the bound on the number of edges can be simplified to $(1-\alpha)\left(\begin{array}{l}n \\ 2\end{array}\right)$ in Corollary 4 .

Suppose constants $A$ and $\alpha>0$ are fixed as in Theorem 7. We need to show that, for all $\beta>0$, there exists a constant $d_{0}=d_{0}(A, \alpha, \beta)>0$ such that every subgraph $G^{\prime} \subseteq G$ with at least $(1-(1-\mathrm{w}(\alpha))(\alpha+\mathrm{w}(\alpha))+\beta) e(G)$ edges has circumference at least $(1-\alpha) n$ if $d=p n \geq d_{0}$, provided the number of vertices in $G$ is sufficiently large.

Let

$$
f(\alpha):=(1-\mathrm{w}(\alpha))(\alpha+\mathrm{w}(\alpha)) .
$$

Observe that for all $0 \leq \delta \leq \alpha \leq 1$, we have

$$
\alpha \leq f(\alpha) \leq 2 \alpha \quad \text { and } \quad f(\alpha)-f(\alpha-\delta) \leq 2 \delta
$$

Suppose the adversary creates a graph $G^{\prime} \subseteq G$ by deleting at most

$$
(f(\alpha)-\beta) e(G)
$$

edges from $G$. Since $G$ is $(p, A)$-uniform, the graph $G^{\prime}$ is $(p, A)$-upper-uniform. We shall prove the existence of a sufficiently long cycle in $G^{\prime}$ in two steps. First, we apply the Regularity Lemma, Lemma 9 to $G^{\prime}$ and conclude from Corollary 4 that there exists a long cycle in the so-called reduced graph. Second, we embed a cycle in $G^{\prime}$ into this structure.

We start off by defining the values of all constants, where we refrain from simplifying certain expressions so as not to obscure them. The particular values of the constants are of less importance, as long as they are independent of $n$. Define

$$
\varrho:=\frac{\beta}{8}, \quad \tau:=\frac{\beta}{8}, \quad \mu:=\frac{\tau}{32} .
$$


Choose $\varepsilon_{0}=\varepsilon(\varrho, \mu)>0$ according to Lemma 10. Suppose that $k_{1}$ is a sufficiently large integer such that Corollary 4 guarantees a cycle of length at least $(1-(\alpha-\tau / 4)) k$ for all graphs on $k \geq k_{1}$ vertices with at least

$$
\left(1-f\left(\alpha-\frac{\tau}{4}\right)+\frac{\tau}{2}\right)\left(\begin{array}{l}
k \\
2
\end{array}\right)
$$

edges. Let

$$
\varepsilon:=\min \left\{\varepsilon_{0}, \frac{1}{7}, \frac{\beta}{32}, \frac{\tau}{32}\right\} \quad \text { and } \quad k_{0}:=\max \left\{\varepsilon^{-1}, k_{1}\right\}
$$

and

$$
\nu:=\frac{1}{2 K_{0}},
$$

where $K_{0}=K_{0}\left(\varepsilon, A, k_{0}\right)$ comes from Lemma 9 . Let $d_{1} \leftarrow d_{0}\left(\varepsilon, A, k_{0}\right)$ be as in Lemma 9 and let $d_{2} \leftarrow d_{0}(\varrho, \mu, \nu, A)$ be as in Lemma 10. We shall prove Theorem 7 with

$$
d_{0}:=\max \left\{d_{1}, d_{2},\left(\frac{8 A}{\nu \beta}\right)^{2}\right\} .
$$

Since $G^{\prime} \subseteq G$ is $(p, A)$-upper-uniform, we apply Lemma 9 to $G^{\prime}$ with parameters $\varepsilon, A$, and $k_{0}$. Thus, we obtain an $\left(\varepsilon, G^{\prime}, p\right)$-regular partition $\Pi=\left(V_{0}, V_{1}, \ldots, V_{k}\right)$ of $V\left(G^{\prime}\right)$ with $k_{0} \leq k \leq K_{0}$. We call a pair $\left(V_{i}, V_{j}\right), 1 \leq i<j \leq k$, regular and $G^{\prime}$-dense if it is $(\varepsilon, p)$-regular and

$$
d_{G^{\prime}, p}\left(V_{i}, V_{j}\right) \geq \varrho .
$$

Consider the reduced graph $R$ in which every vertex corresponds to a class $V_{i}, 1 \leq$ $i \leq k$, and two vertices $i$ and $j$ are connected if and only if $\left(V_{i}, V_{j}\right)$ is regular and $G^{\prime}$-dense. Our aim is to show a lower bound on the number of edges in $R$.

Let $m:=\left|V_{1}\right|=\left|V_{2}\right|=\ldots=\left|V_{k}\right|$ and observe that $\nu n \leq m \leq n / k$. We need to take into account four different types of edges in $G^{\prime}$.

(i) Due to (2) and (3), the number of edges in $G^{\prime}$ that are incident to the vertices in $V_{0}$ is

$$
\begin{aligned}
e_{G^{\prime}}\left(V_{0}\right)+e_{G^{\prime}}\left(V_{0}, V \backslash V_{0}\right) & \leq p\left(\begin{array}{c}
\varepsilon n \\
2
\end{array}\right)+A \sqrt{d} \varepsilon n+p \varepsilon(1-\varepsilon) n^{2}+A \sqrt{d \varepsilon} n \\
& \leq\left(\frac{\varepsilon^{2}}{2}+\varepsilon(1-\varepsilon)\right) p n^{2}+2 A \sqrt{d \varepsilon} n \\
& \leq \varepsilon p n^{2}+2 A \sqrt{d \varepsilon} n \\
& =2 \varepsilon\left(1+\frac{2 A \sqrt{d}}{p n \sqrt{\varepsilon}}\right) \frac{p n^{2}}{2}=2 \varepsilon\left(1+\frac{2 A}{\sqrt{d \varepsilon}}\right) \frac{p n^{2}}{2} .
\end{aligned}
$$

the EleCtronic Journal of COMbinatorics 15 (2008), \#R32 
(ii) The number of edges that belong to irregular pairs is

$$
\leq \varepsilon\left(\begin{array}{l}
k \\
2
\end{array}\right)\left(p m^{2}+A \sqrt{d} m\right) \leq \varepsilon\left(1+\frac{A k}{\sqrt{d}}\right) \frac{p n^{2}}{2} .
$$

(iii) The number of edges that belong to regular pairs that fail (10) is

$$
<\left(\begin{array}{l}
k \\
2
\end{array}\right) \varrho p m^{2} \leq \varrho \frac{p n^{2}}{2} .
$$

(iv) The number of edges whose endpoints belong to the same $V_{i}, 1 \leq i \leq k$, is

$$
\leq k\left(p\left(\begin{array}{c}
m \\
2
\end{array}\right)+A \sqrt{d} m\right) \leq\left(\frac{1}{k}+\frac{2 A}{\sqrt{d}}\right) \frac{p n^{2}}{2} .
$$

Summing up the estimates (11)-(14), we conclude that the number of edges in $G^{\prime}$ that are not contained in regular and $G^{\prime}$-dense pairs is

$$
\begin{aligned}
& <\left(2 \varepsilon\left(1+\frac{2 A}{\sqrt{d \varepsilon}}\right)+\varepsilon\left(1+\frac{A k}{\sqrt{d}}\right)+\varrho+\frac{1}{k}+\frac{2 A}{\sqrt{d}}\right) \frac{p n^{2}}{2} \\
& \leq\left(4 \varepsilon+\varrho+\frac{A}{\sqrt{d}}(4 \sqrt{\varepsilon}+\varepsilon k+2)\right) \frac{p n^{2}}{2} \leq\left(4 \varepsilon+\varrho+\frac{A k}{\sqrt{d}}\right) \frac{p n^{2}}{2} .
\end{aligned}
$$

We want to show that

$$
e(R)>(1-f(\alpha)+\tau)\left(\begin{array}{l}
k \\
2
\end{array}\right)
$$

For the sake of contradiction, suppose $R$ has at most these many edges. Then the number of edges in regular and $G^{\prime}$-dense pairs in $G^{\prime}$ is

$$
\leq(1-f(\alpha)+\tau)\left(\begin{array}{l}
k \\
2
\end{array}\right)\left(p m^{2}+A \sqrt{d} m\right) \leq\left(1-f(\alpha)+\tau+\frac{A k}{\sqrt{d}}\right) \frac{p n^{2}}{2} .
$$

Hence, adding (15) and (17), we have

$$
\begin{aligned}
e\left(G^{\prime}\right) & <\left(1-f(\alpha)+\tau+4 \varepsilon+\varrho+\frac{2 A k}{\sqrt{d}}\right) \frac{p n^{2}}{2} \\
& \leq\left(1-f(\alpha)+\frac{\beta}{8}+\frac{\beta}{8}+\frac{\beta}{8}+\frac{A}{\nu \sqrt{d}}\right) \frac{p n^{2}}{2} \\
& \leq\left(1-f(\alpha)+\frac{\beta}{2}\right) \frac{p n^{2}}{2} .
\end{aligned}
$$

the EleCtronic journal of COMbinatorics 15 (2008), \#R32 
On the other hand, since the enemy may not delete more than an $(f(\alpha)-\beta)$ fraction of the edges in $G$, we can derive that

$$
\begin{aligned}
e\left(G^{\prime}\right) & \geq(1-f(\alpha)+\beta)\left(p\left(\begin{array}{l}
n \\
2
\end{array}\right)-A \sqrt{d} n\right) \\
& =(1-f(\alpha)+\beta)\left(1-\frac{1}{n}-\frac{2 A}{\sqrt{d}}\right) \frac{p n^{2}}{2} \\
& \geq(1-f(\alpha)+\beta)\left(1-\frac{3 A}{\sqrt{d}}\right) \frac{p n^{2}}{2} \\
& \geq\left(1-f(\alpha)+\beta-\frac{4 A}{\sqrt{d}}\right) \frac{p n^{2}}{2} \geq\left(1-f(\alpha)+\frac{\beta}{2}\right) \frac{p n^{2}}{2} .
\end{aligned}
$$

This contradicts (18) and hence (16) must hold. Combining (16) and (9) we conclude

$$
e(R)>(1-f(\alpha)+\tau)\left(\begin{array}{l}
k \\
2
\end{array}\right) \geq\left(1-f\left(\alpha-\frac{\tau}{4}\right)+\frac{\tau}{2}\right)\left(\begin{array}{l}
k \\
2
\end{array}\right)
$$

Applying Corollary 4 to $R$, we conclude that, by our choice of $k_{0}, R$ contains a cycle of length at least $(1-\alpha+\tau / 4) k$.

Starting with the long cycle in the reduced graph, let us now embed a cycle into the original graph $G^{\prime}$. Let $C_{t}$ denote the cycle in the reduced graph $R$ of length $t \geq(1-\alpha+\tau / 4) k$. The entire construction is illustrated in Figure 1 on the following page. W.l.o.g. $C_{t}=\left(V_{1}, V_{2}, \ldots, V_{t}\right)$. We embed a cycle of length at least $(1-\alpha) n$ into $G^{\prime}$ as follows. For all $1 \leq i \leq\lfloor t / 2\rfloor$, we simultaneously apply Lemma 10 to each pair $\left(V_{2 i-1}, V_{2 i}\right)$. This yields sets $X_{2 i-1} \subseteq V_{2 i-1}$ and $X_{2 i} \subseteq V_{2 i},\left|X_{2 i-1}\right|=\left|X_{2 i}\right|=\varepsilon m$, such that every pair of vertices $\left(x_{2 i-1}, x_{2 i}\right) \in\left(X_{2 i-1}, X_{2 i}\right)$ is connected by a path of length at least $2(1-2 \mu) m$ using edges from $G^{\prime}\left[X_{2 i-1}, X_{2 i}\right]$. We can connect these paths by putting an arbitrary edge between $X_{2 i}$ and $X_{2 i+1}$ for all $1 \leq i<\lfloor t / 2\rfloor$. Note that

$$
e_{G^{\prime}}\left(X_{2 i}, X_{2 i+1}\right) \geq\left(d_{2 i, 2 i+1}-\varepsilon\right) p(\varepsilon m)^{2} \geq 1
$$

since $\left(V_{2 i}, V_{2 i+1}\right)$ is $(\varepsilon, p)$-regular with density $d_{2 i, 2 i+1} \geq \varrho$.

If $t$ is even, we close the cycle by putting an arbitrary edge between $X_{1}$ and $X_{t}$. Otherwise we connect $X_{1}$ and $X_{t-1}$ through one vertex in $V_{t}$. Clearly, there are at least $(1-2 \varepsilon) m$ vertices in $V_{t}$ that are adjacent to some vertex in $X_{1}$ as well as some vertex in $X_{t-1}$. 


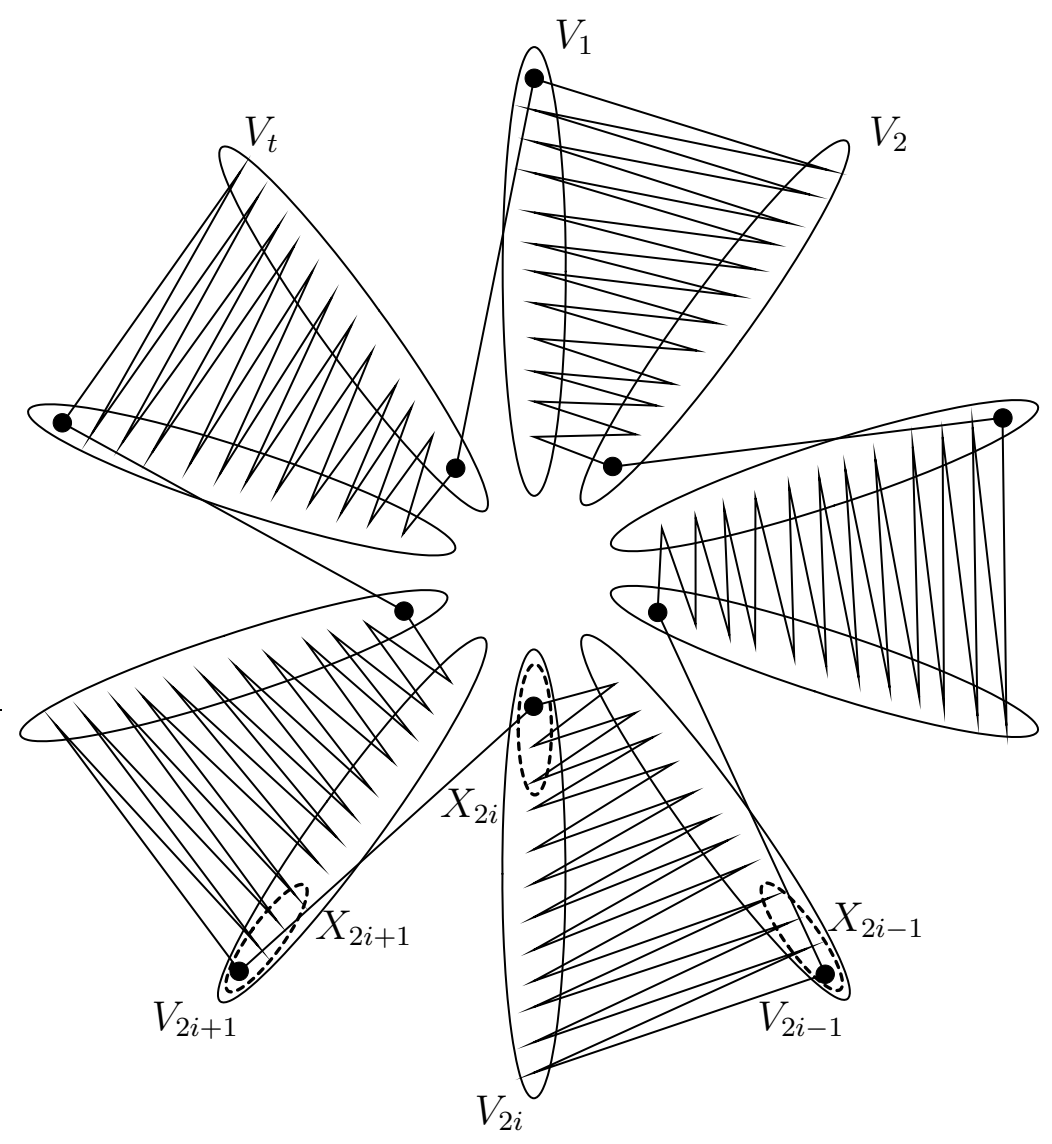

Figure 1: Embedding of a long cycle into $G^{\prime}$. We suppose $1, \ldots, t$ form a cycle in the reduced graph.

Thus, for $n$ sufficiently large, we have constructed a cycle of length at least

$$
\begin{aligned}
\left\lfloor\frac{(1-\alpha+\tau / 4) k}{2}\right\rfloor \cdot 2(1-2 \mu) m & \geq((1-\alpha+\tau / 4) k-2) \cdot(1-2 \mu) m \\
& \geq\left(1-\alpha+\tau / 4-\frac{2}{k}\right) k(1-2 \mu)(1-\varepsilon) \frac{n}{k} \\
& \geq(1-\alpha+\tau / 4-2 \varepsilon)(1-2 \mu)(1-\varepsilon) n \\
& \geq(1-\alpha+\tau / 4-4 \varepsilon-2 \mu) n>(1-\alpha) n .
\end{aligned}
$$

This concludes the proof of Theorem 7 . 


\subsection{Proof of Theorem 8}

As in Section 3.1, we need to show both an upper and a lower bound on the local resilience $r_{l}$ of $G$ w.r.t. containing long cycles.

\subsubsection{Proof of the upper bound for $r_{l}$}

The upper bound is shown by providing an appropriate strategy so that the adversary can destroy all cycles of length at least $(1-\alpha) n$ in any $(p, A)$-uniform graph $G$ by removing no more than $(1 / 2+\beta) \operatorname{deg}(v)$ edges at each vertex $v$ for some arbitrarily small constant $\beta>0$. His strategy is to find an approximately even bipartition of $V(G)$ such that each vertex $v$ has at most $(1 / 2+\beta) \operatorname{deg}(v)$ neighbors in the other partition class. In what follows, we deal with technical details and calculations regarding such bipartition: the adversary may start by randomly paritioning the vertex set and then must move some vertices which might have low degree from one part to the other without (significantly) affecting other vertices.

In the first step, we omit all vertices of very small or very large degree in $G$. The following claim, which is a simple consequence of $(p, A)$-regularity, states that there are only very few of those. The proof is a straightforward application of the definition of $(p, A)$-regularity; the details are left to the reader.

Claim 14. Let $A, \delta$, and $\nu$ be positive constants. Then there exists a constant $d_{0}=$ $d_{0}(A, \delta, \nu)$ such that every sufficiently large $(p, A)$-uniform graph $G$ on $n$ vertices with $d=p n \geq d_{0}$ satisfies the following property: The number of vertices $v \in V(G)$ with

$$
|\operatorname{deg}(v)-d|>\delta d
$$

is at most $\nu$.

We next we show that a bipartition in which almost all vertices in one part satisfy a maximum degree condition can be adapted so that a slightly weaker degree condition holds for all vertices.

Claim 15. For all $\beta>0$, there exists a positive constant $\nu_{\beta}$ such that, for all $A>0$ and $0<\nu \leq \nu_{\beta}$, there exists a constant $d_{0}=d_{0}(A, \nu)$ such that, for $n$ sufficiently large, every $(p, A)$-upper-uniform graph $G$ on $n$ vertices with $d=p n \geq d_{0}$ satisfies the following property: Suppose $V=X \cup Y$ is a partition of $V$ such that at most $\nu$ n vertices $x \in X$ violate

$$
|\Gamma(x) \cap Y| \leq\left(\frac{1}{2}+\frac{\beta}{2}\right) \operatorname{deg}(x) .
$$

THE ELECTRONiC JOURNAL OF COMBINATORICS 15 (2008), \#R32 
Then there exists a set $X^{\prime} \subseteq X$ of size at most $4 \nu n$ such that, for all vertices $x \in$ $X \backslash X^{\prime}$, we have

$$
\left|\Gamma(x) \cap\left(Y \cup X^{\prime}\right)\right| \leq\left(\frac{1}{2}+\beta\right) \operatorname{deg}(x)
$$

and, for all $x^{\prime} \in X^{\prime}$, we have

$$
\left|\Gamma\left(x^{\prime}\right) \cap\left(X \backslash X^{\prime}\right)\right| \leq \frac{1}{2} \operatorname{deg}(x) .
$$

Proof. First, set $d_{0}$ sufficiently large so that, according to Claim 14, at most $\nu n$ vertices in $G$ have total degree less than $d / 2$. Now, consider the following process, which inductively constructs a sequence of sets $X_{0} \subseteq X_{1} \subseteq \ldots \subseteq X_{t}$. We start with

$$
X_{0}:=\left\{x \in X:|\Gamma(x) \cap Y|>\left(\frac{1}{2}+\frac{\beta}{2}\right) \operatorname{deg}(x)\right\} .
$$

At step $i$, we add some vertex $x_{i}$ from $X \backslash X_{i-1}$ that satisfies

$$
\left|\Gamma\left(x_{i}\right) \cap\left(Y \cup X_{i-1}\right)\right|>\left(\frac{1}{2}+\beta\right) \operatorname{deg}\left(x_{i}\right)
$$

to $X_{i-1}$, forming set $X_{i}$. The process terminates at time $t$ with $X^{\prime}:=X_{t}$ when no such vertex exists. Clearly, once this process stops, all vertices in $X^{\prime}$ satisfy the desired degree conditions. We shall show that $\left|X^{\prime}\right| \leq 4 \nu n$, i.e., the process stops at time $t \leq 3 \nu n$.

For the sake of contradiction, suppose that the process reaches time $t_{0}=3 \nu n$. Observe that in each step $i, 1 \leq i \leq t_{0}$, the new vertex $x_{i}$ satisfies

$$
\left|\Gamma\left(x_{i}\right) \cap Y\right| \leq\left(\frac{1}{2}+\frac{\beta}{2}\right) \operatorname{deg}\left(x_{i}\right)
$$

by definition of $X_{0}$. Consequently, we have

$$
\left|\Gamma\left(x_{i}\right) \cap X_{i-1}\right|=\left|\Gamma\left(x_{i}\right) \cap\left(Y \cup X_{i-1}\right)\right|-\left|\Gamma\left(x_{i}\right) \cap Y\right|>\frac{\beta}{2} \operatorname{deg}\left(x_{i}\right) .
$$

This yields the following lower bound on the number of edges in $X_{t_{0}}$ :

$$
e\left(X_{t_{0}}\right) \geq \sum_{i=1}^{t_{0}}\left|\Gamma\left(x_{i}\right) \cap X_{i-1}\right|>\sum_{i=1}^{t_{0}} \frac{\beta}{2} \operatorname{deg}\left(x_{i}\right)
$$

the EleCtronic Journal of COMbinatorics 15 (2008), \#R32 
and, since there are at most $\nu n$ vertices of degree less than $d / 2$ in $X$,

$$
>\left(t_{0}-\nu n\right) \cdot \frac{\beta}{4} d=\frac{\beta}{2} \nu d n .
$$

On the other hand, since $G$ is $(p, A)$-upper-uniform, we have

$$
e\left(X_{t_{0}}\right) \leq p\left(\begin{array}{c}
\left|X_{t_{0}}\right| \\
2
\end{array}\right)+A \sqrt{d}\left|X_{t_{0}}\right| \leq 8 \nu^{2} d n\left(1+\frac{A}{2 \nu \sqrt{d}}\right)
$$

and, setting $d_{0} \geq(A /(2 \nu))^{2}$ and $\nu_{\beta}:=\beta / 32$,

$$
\leq 16 \nu^{2} d n \leq 16 \nu_{\beta} \nu d n=\frac{\beta}{2} \nu d n
$$

This yields a contradiction and completes the proof of Claim 15.

Continuing the proof of the upper bound, suppose $\beta>0$ and $A>0$ are fixed. Applying Claim 15 with parameter $\beta$ yields constant $\nu_{\beta}$. Let

$$
\nu:=\min \left\{\frac{1-2 \alpha}{10}, \nu_{\beta}\right\}
$$

Invoking Claim 15 with parameters $A$ and $\nu$ yields a constant $d_{1} \leftarrow d_{0}$. Plugging parameters $A, \delta \leftarrow 1 / 2$ and $\nu \leftarrow \nu / 2$ into Claim 14 yields another constant $d_{2} \leftarrow d_{0}$. Choose $d_{0}$ as the maximum of both $d_{1}$ and $d_{2}$. Then, owing to Claim 14, every sufficiently large $(p, A)$-uniform graph on $n$ vertices with $d=p n \geq d_{0}$ contains at most $(\nu / 2) n$ vertices of degree less then $d / 2$. We call these vertices thin and the other ones normal.

Consider a random bipartition of $V(G)$ by tossing a fair coin for each vertex. Let $\Delta$ be the random variable counting the number of vertices by which the larger of both partition classes exceeds $n / 2$. Clearly, for $n$ sufficiently large, the probability that $\Delta$ is at most $(\nu / 2) n$ is, say, at least 0.6. Assuming that $d_{0}$ is sufficiently large, a standard application of Chernoff's and Markov's inequalities yields that, with at least the same probability, among the normal vertices there are at most $(\nu / 2) n$ vertices $v$ that have at least $(1 / 2+\beta / 2) \operatorname{deg}(v)$ neighbors in the other partition class. Since the conjunction of those two events has positive probability, there exists a bipartition of $V(G)$ that satisfies both. Fix any such partition $V(G)=X \cup Y$. Our next aim is to adjust this partition slightly so that every vertex has at most $(1 / 2+\beta) \operatorname{deg}(v)$ in the other partition class. 
Call a vertex that has more than $(1 / 2+\beta / 2) \operatorname{deg}(v)$ neighbors in the other partition class unhappy. Clearly, the total number of unhappy vertices is bounded from above by the sum of thin vertices and normal ones that violate this degree condition. By our choice of $X$ and $Y$, this is bounded from above by $\nu n$. Hence, Claim 15 asserts that there exists a set $X^{\prime}$ of size at most $4 \nu n$ such that, after shifting all vertices of $X^{\prime}$ into $Y$, all the remaining vertices $x \in X \backslash X^{\prime}$ satisfy

$$
\left|\Gamma(x) \cap\left(Y \cup X^{\prime}\right)\right| \leq\left(\frac{1}{2}+\beta\right) \operatorname{deg}(x),
$$

and, for all vertices $x^{\prime} \in X^{\prime}$, we have

$$
\left|\Gamma\left(x^{\prime}\right) \cap\left(X \backslash X^{\prime}\right)\right| \leq \frac{1}{2} \operatorname{deg}(x) .
$$

Now, all vertices that were initially in $X$ satisfy our desired degree bound w.r.t. the new partition. Observe that shifting the set $X^{\prime}$ from $X$ to $Y$ cannot make any vertex of $Y$ unhappy that was not already unhappy before. Hence, we can apply Claim 15 to the new partition $\widehat{X}:=X \backslash X^{\prime}$ and $\widehat{Y}:=Y \cup X^{\prime}$ to obtain a set $Y^{\prime} \subseteq \widehat{Y}$ such that partitioning $V(G)$ into $\widehat{X} \cup Y^{\prime}=\left(X \backslash X^{\prime}\right) \cup Y^{\prime}$ and $\widehat{Y} \backslash Y^{\prime}=\left(Y \cup X^{\prime}\right) \backslash Y^{\prime}$ yields that, for all vertices $v \in V(G)$, the number of neighbors in the other class is at most

$$
\left(\frac{1}{2}+\beta\right) \operatorname{deg}(v) \text {. }
$$

By deleting all edges with endpoints in distinct partition classes, we destroy all cycles of length at least $\frac{1}{2} n+\Delta+4 \nu n \leq(1-\alpha) n$, while no vertex loses more edges than allowed. This proves the upper bound for $r_{l}$ in Theorem 8 .

\subsubsection{Proof of the lower bound for $r_{l}$}

We give the proof of the lower bound in Theorem 8 in this section. Suppose $G$ is a $(p, A)$-uniform graph as in Theorem 8 , and let $\alpha$ and $\beta>0$ be fixed. Suppose the adversary removed from $G$ the edges of a subgraph $H$ with degree at most $(1 / 2-$ $\beta) \operatorname{deg}_{G}(v)$ at any vertex $v$, which resulted in a $(p, A)$-upper-uniform graph $G^{\prime}$. We shall show that, no matter how $H$ was chosen, $G^{\prime}$ contains a cycle of length $(1-\alpha) n$, provided that $n$ is sufficiently large. The main approach to achieve this is similar to the approach that we pursued in Section 3.1.2. We apply the Regularity Lemma to $H$, find an appropriate cycle in the reduced graph, and then use Lemma 10 to construct a long cycle in $H$. The main difference is that we now need the cycle in the reduced graph to cover almost all vertices instead of just a constant fraction of 
the vertices. The proof of Theorem 8 is thus readily at hand when we establish the following lemma.

Lemma 16. Let $A, \beta>0$, and $\kappa>0$ be fixed. There exist positive constants $\varrho=$ $\varrho(\beta)$ and $\varepsilon=\varepsilon(\beta, \kappa)$ such that, for all $K_{0}>0$ and $n$ sufficiently large, there exists $d_{0}=d_{0}\left(A, \beta, \kappa, K_{0}\right)$ such that the following assertion holds. Suppose $d=p n \geq d_{0}$ and $G^{\prime}$ was obtained by removing the edge set of a graph $H$ such that

$$
\operatorname{deg}_{H}(v) \leq\left(\frac{1}{2}-\beta\right) \operatorname{deg}_{G}(v)
$$

for all $v \in V(G)$, where $G$ is a $(p, A)$-uniform graph on $n$ vertices, as in Theorem 8 . Let $\left(V_{0}, V_{1}, \ldots, V_{k}\right)$ be a $(\varepsilon, p)$-regular partition of $G^{\prime}$ with $\varepsilon^{-1} \leq k \leq K_{0}$. Consider the reduced graph $R$ on the vertex set $[k]$, which contains an edge $\{i, j\}, 1 \leq i, j \leq k$, if and only if $\left(V_{i}, V_{j}\right)$ is $(\varepsilon, p)$-regular and satisfies

$$
e_{G^{\prime}}\left(V_{i}, V_{j}\right) \geq \varrho p\left|V_{i}\right|\left|V_{j}\right|
$$

Then $R$ contains an even cycle of length at least $(1-\kappa) k$.

Let us continue with the proof of the lower bound in Theorem 8 . Let $\alpha>0$ be as given in that theorem and let $\beta>0$ be fixed. By an application of Lemma 16 with parameters

$$
\beta \quad \text { and } \quad \kappa:=\frac{\alpha}{3},
$$

we obtain constants $\varrho$ and $\varepsilon_{1} \leftarrow \varepsilon$. Next we apply Lemma 10 with

$$
\varrho \quad \text { and } \quad \mu:=\frac{\alpha}{6}
$$

which yields $\varepsilon_{2} \leftarrow \varepsilon$. Recall that $G^{\prime} \subseteq G$ is $(p, A)$-upper-uniform. We fix

$$
\varepsilon:=\min \left\{\varepsilon_{1}, \varepsilon_{2}, \frac{\alpha}{4}\right\}
$$

and plug

$$
\varepsilon, \quad k_{0}:=\varepsilon^{-1}, \quad \text { and } A
$$

into the Regularity Lemma, Lemma 9, in order to fix constants $K_{0}, n_{0}$, and $d_{1} \leftarrow d_{0}$. Finally, setting

$$
\nu:=\frac{1-\varepsilon}{K_{0}}
$$

THE ELECTRONiC JOURnAl of COMBInAtorics 15 (2008), \#R32 
in Lemma 10 yields $d_{2} \leftarrow d_{0}$, and plugging $K_{0}$ into Lemma 16 yields $d_{3} \leftarrow d_{0}$. Thus, we fix $d_{0}$ as

$$
d_{0}:=\max \left\{d_{1}, d_{2}, d_{3}\right\} .
$$

With the choice of constants above, we can show that there exists a sufficiently long cycle in $G^{\prime}$ along the same lines as in the proof of Theorem 7. Owing to Lemma 9, $G^{\prime}$ admits an $\left(\varepsilon, G^{\prime}, p\right)$-regular partition $\left(V_{0}, V_{1}, \ldots, V_{k}\right)$ with $\varepsilon^{-1} \leq k \leq K_{0}$. Then Lemma 16 asserts that there exists an even cycle of length $k^{\prime} \geq(1-\kappa) k$ in the reduced graph $R$. Now applying Lemma 10 to every second pair of that cycle and connecting the generated paths in those pairs by an arbitrary edge, we create a long cycle in $G^{\prime}$ as depicted in Figure 1. Let $m$ denote the size of the partition classes $V_{i}$, $1 \leq i \leq k$. This cycle has length at least

$$
\begin{aligned}
\frac{k^{\prime}}{2} \cdot 2(1-2 \mu) m & \geq(1-\kappa) k(1-2 \mu) \frac{(1-\varepsilon) n}{k} \\
& =(1-\kappa)(1-2 \mu)(1-\varepsilon) n \geq(1-\alpha) n+1
\end{aligned}
$$

by our choice of $\kappa, \varepsilon$, and $\mu$. This concludes the proof of the lower bound for $r_{l}$ in Theorem 8. We now prove Lemma 16.

Proof of Lemma 16. We start by proving a general fact about graphs on $k$ vertices that are almost complete.

Claim 17. Let $\kappa$ be a positive constant. Then there exists $\varepsilon>0$ such that any graph on $k \geq \varepsilon^{-1}$ vertices with at least

$$
(1-\varepsilon)\left(\begin{array}{l}
k \\
2
\end{array}\right)
$$

edges satisfies the following property: There exists a subgraph on an even number of vertices $k^{\prime} \geq(1-\kappa) k$ with minimum degree at least $(1-2 \kappa) k$.

Proof. For simplicity we assume that $\kappa k$ is integral. The general case follows easily along the same lines and is left to the reader. Let $\varepsilon:=\kappa^{2} / 2$. Suppose $K$ is a graph on the vertex set $\left\{v_{1}, \ldots, v_{k}\right\}$ such that

$$
\operatorname{deg}\left(v_{1}\right) \geq \operatorname{deg}\left(v_{2}\right) \geq \ldots \geq \operatorname{deg}\left(v_{k}\right) .
$$

Let $k^{\prime}=(1-\kappa) k+1$ and $K^{\prime}$ be the subgraph of $K$ induced by the vertices $\left\{v_{1}, \ldots, v_{k^{\prime}}\right\}$. If, for all $1 \leq i \leq k^{\prime}$, we have

$$
\operatorname{deg}_{K^{\prime}}\left(v_{i}\right) \geq(1-3 \kappa / 2) k,
$$


then we are home. Note that even if we need to remove, say, the last vertex from $K^{\prime}$ so that the number of vertices in the chosen graph $K^{\prime \prime} \subset K^{\prime}$ is even, we would have

$$
\operatorname{deg}_{K^{\prime \prime}}\left(v_{i}\right) \geq \operatorname{deg}_{K^{\prime}}\left(v_{i}\right)-1 \geq(1-3 \kappa / 2-1 / k) k \geq(1-3 \kappa / 2-\varepsilon) k \geq(1-2 \kappa) k,
$$

for all $1 \leq i<k^{\prime}$. Hence, suppose for the sake of contradiction that there exists an index $j, 1 \leq j \leq k^{\prime}$, such that

$$
\operatorname{deg}_{K^{\prime}}\left(v_{j}\right)<(1-3 \kappa / 2) k
$$

This implies that, for all $j \leq \ell \leq k$, we have

$$
\operatorname{deg}_{K}\left(v_{l}\right) \leq \operatorname{deg}_{K}\left(v_{j}\right) \leq \operatorname{deg}_{K^{\prime}}\left(v_{j}\right)+\left(k-k^{\prime}\right)<(1-\kappa / 2) k-1 .
$$

Thus, the number of edges in $K$ is less than

$$
\left(\begin{array}{l}
k \\
2
\end{array}\right)-\frac{1}{4} \sum_{\ell \geq j} \kappa k \leq\left(\begin{array}{l}
k \\
2
\end{array}\right)-\frac{1}{4} \kappa k \cdot\left(k-k^{\prime}+1\right) \leq\left(1-\kappa^{2} / 2\right)\left(\begin{array}{l}
k \\
2
\end{array}\right) .
$$

This, however, is a contradiction to $e(K) \geq(1-\varepsilon)\left(\begin{array}{l}k \\ 2\end{array}\right)$.

The next claim is a simple consequence of $(p, A)$-uniformity; the calculations are left to the reader.

Claim 18. Let $A, \delta$, and $\nu$ be positive constants. Then there exists a constant $d_{0}=$ $d_{0}(A, \delta, \nu)$ such that every sufficiently large $(p, A)$-uniform graph $G$ on $n$ vertices with $d=p n \geq d_{0}$ satisfies the following property: For any disjoint sets $U \subseteq V(G)$ with $|U| \geq \nu n$ and $W \subseteq V(G)$ with $|W| \geq n / 4$, the number of vertices $u \in U$ that satisfy

$$
\left|\Gamma_{G}(u) \cap W\right| \geq(1-\delta) p|W|
$$

is at least $\frac{3}{4}|U|$.

Lemma 16 can be derived from Claims 14, 17, and 18 as follows. Let $\beta$ and $\kappa$ be as given in the statement of Lemma 16. Let

$$
\varrho:=\frac{\beta}{4} \text { and } \quad \gamma:=\frac{\beta}{4}
$$

and apply Claim 17 with parameter $\kappa \leftarrow \min \{\kappa, \gamma / 2\}$ in order to obtain $\varepsilon_{1} \leftarrow \varepsilon$. Set

$$
\varepsilon:=\min \left\{\varepsilon_{1}, \frac{\beta}{4}\right\}, \quad \delta:=\frac{\beta}{8}, \quad \text { and } \quad \nu:=\frac{1-\varepsilon}{K_{0}},
$$


and apply Claim 14 with parameters $\delta$ and $\nu \leftarrow \nu / 4$, which yields $d_{1} \leftarrow d_{0}$. Moreover, apply Claim 18 with parameters $\delta$ and $\nu$, which yields $d_{2} \leftarrow d_{0}$. Define $d_{0}$ as the maximum of $d_{1}$ and $d_{2}$. Claim 17 asserts that there is a graph $R^{\prime}$ on $k^{\prime} \geq(1-\kappa) k$ vertices such that $V\left(R^{\prime}\right) \subseteq[k]$, the minimum degree of $R^{\prime}$ is at least $(1-\gamma) k$, and each edge $\{i, j\}$ of $R^{\prime}$ implies that the pair $\left(V_{i}, V_{j}\right)$ is $(\varepsilon, p)$-regular in $G^{\prime}$. Moreover, the number of vertices in $R^{\prime}$ is even. Let $R \subseteq R^{\prime}$ be a graph on the same vertex set that contains an edge $\{i, j\}$ if and only if $\{i, j\} \in E\left(R^{\prime}\right)$ and

$$
e_{G^{\prime}}\left(V_{i}, V_{j}\right) \geq \varrho p\left|V_{i}\right|\left|V_{j}\right| \text {. }
$$

Owing to Dirac's theorem, it remains to show that $R$ has minimum degree $k / 2 \geq k^{\prime} / 2$ so as to assert the existence of a Hamiltonian cycle in $R$.

For the sake of contradiction, suppose that there exists a vertex $r \in V(R)$ that has degree less than $k / 2$. Let $m$ denote the size of the partition classes $V_{i}, 1 \leq i \leq k$. Owing to Claim 14, there are at most $m / 4$ vertices $v \in V_{r}$ with $\operatorname{deg}_{G}(v)>(1+$ $\delta) p n$. Consider the vertex set $W_{r} \subseteq V\left(G^{\prime}\right)$ spanned by the partition classes $V_{j}$ that correspond to regular, but sparse edges $\{r, j\}$ in $R^{\prime}$, i.e.,

$$
\begin{aligned}
E_{r} & :=\left\{\{r, j\} \in E\left(R^{\prime}\right): e_{G^{\prime}}\left(V_{r}, V_{j}\right)<\varrho p\left|V_{r}\right|\left|V_{j}\right|\right\}, \\
W_{r} & :=\bigcup_{\{r, j\} \in E_{r}} V_{j} .
\end{aligned}
$$

Note that, crucially, we have $\left|E_{r}\right| \geq(1-\gamma) k-k / 2=(1 / 2-\gamma) k$ and thus

$$
\left|W_{r}\right|=\left|E_{r}\right| m \geq(1 / 2-\gamma) k m \geq(1 / 2-\gamma)(1-\varepsilon) n \geq \frac{n}{4},
$$

assuming that $\beta \leq 1 / 2$. Hence, we can apply Claim 18 to $G$ with $U \leftarrow V_{r}$ and $W \leftarrow$ $W_{r}$ and conclude that there is a set $V_{r}^{\prime} \subseteq V_{r}$ of size at least $m / 2$ such that each $v \in V_{r}^{\prime}$ satisfies

$$
\operatorname{deg}_{G}(v) \leq(1+\delta) p n \quad \text { and } \quad\left|\Gamma_{G}(v) \cap W_{r}\right| \geq(1-\delta) p\left|W_{r}\right|,
$$

that is, each of those vertices has bounded total degree and approximately the the right proportion of neighbors in the set $W_{r}$ before the deletion takes place. Owing to the definition of $W_{r}$, we have

$$
e_{G^{\prime}}\left(V_{r}, W_{r}\right)<\left|E_{r}\right| \varrho p\left|V_{r}\right|\left|V_{j}\right|=\varrho p m\left|W_{r}\right| .
$$

Furthermore, $e_{G^{\prime}}\left(V_{r}^{\prime}, W_{r}\right) \leq e_{G^{\prime}}\left(V_{r}, W_{r}\right)$ holds trivially. Thus, by the averaging principle, there must be a vertex $v_{r} \in V_{r}^{\prime}$ that satisfies

$$
\left|\Gamma_{G^{\prime}}\left(v_{r}\right) \cap W_{r}\right| \leq \frac{e_{G^{\prime}}\left(V_{r}^{\prime}, W_{r}\right)}{\left|V_{r}^{\prime}\right|}<2 \varrho p\left|W_{r}\right| .
$$


This, however, implies that

$$
\begin{aligned}
\operatorname{deg}_{H}\left(v_{r}\right) & \geq\left|\Gamma_{G}\left(v_{r}\right) \cap W_{r}\right|-\left|\Gamma_{G^{\prime}}\left(v_{r}\right) \cap W_{r}\right| \\
& >(1-\delta-2 \varrho) p\left|W_{r}\right| \\
& \geq(1-\delta-2 \varrho) p(1 / 2-\gamma)(1-\varepsilon) n \\
& \geq(1 / 2-(\delta+\varrho+\gamma+\varepsilon)) p n \\
& \geq \frac{1 / 2-(\delta+\varrho+\gamma+\varepsilon)}{1+\delta} \operatorname{deg}_{G}\left(v_{r}\right) \\
& \geq(1 / 2-(2 \delta+\varrho+\gamma+\varepsilon)) \operatorname{deg}_{G}\left(v_{r}\right) \\
& \geq(1 / 2-\beta) \operatorname{deg}_{G}\left(v_{r}\right),
\end{aligned}
$$

which violates the maximum number of edges that the adversary is allowed to remove at vertex $v_{r}$. This contradiction shows that $R$ satisfies Dirac's condition, and hence $R$ is indeed Hamiltonian, and the proof of Lemma 16 is complete.

\section{References}

[1] B. Bollobás. Extremal graph theory, volume 11 of London Mathematical Society Monographs. Academic Press Inc. [Harcourt Brace Jovanovich Publishers], London, 1978.

[2] B. Bollobás. Extremal graph theory. Dover Publications Inc., Mineola, NY, 2004. Reprint of the original edition from 1978.

[3] J. A. Bondy. Extremal problems of Paul Erdős on circuits in graphs. In Paul Erdös and his mathematics, II (Budapest, 1999), volume 11 of Bolyai Soc. Math. Stud., pages 135-156. János Bolyai Math. Soc., Budapest, 2002.

[4] L. Caccetta and K. Vijayan. Maximal cycles in graphs. Discrete Math., 98(1):17, 1991.

[5] G. A. Dirac. Some theorems on abstract graphs. Proc. London Math. Soc. (3), 2:69-81, 1952.

[6] J. Donadelli, P. E. Haxell, and Y. Kohayakawa. A note on the size-Ramsey number of long subdivisions of graphs. Theor. Inform. Appl., 39(1):191-206, 2005.

[7] P. Erdős and T. Gallai. On maximal paths and circuits of graphs. Acta Math. Acad. Sci. Hungar, 10:337-356 (unbound insert), 1959.

[8] A. Frieze and M. Krivelevich. On two Hamilton cycle problems in random graphs. Israel Journal of Mathematics. To appear. 
[9] S. Gerke and A. Steger. The sparse regularity lemma and its applications. In Surveys in combinatorics 2005, volume 327 of London Math. Soc. Lecture Note Ser., pages 227-258. Cambridge Univ. Press, Cambridge, 2005.

[10] Stefanie Gerke, Yoshiharu Kohayakawa, Vojtěch Rödl, and Angelika Steger. Small subsets inherit sparse $\varepsilon$-regularity. J. Combin. Theory Ser. B, 97(1):3456, 2007.

[11] P. E. Haxell. Partitioning complete bipartite graphs by monochromatic cycles. J. Combin. Theory Ser. B, 69(2):210-218, 1997.

[12] P. E. Haxell, Y. Kohayakawa, and T. Łuczak. The induced size-Ramsey number of cycles. Combin. Probab. Comput., 4(3):217-239, 1995.

[13] P. E. Haxell, Y. Kohayakawa, and T. Łuczak. Turán's extremal problem in random graphs: forbidding even cycles. J. Combin. Theory Ser. B, 64(2):273287, 1995.

[14] P. E. Haxell, Y. Kohayakawa, and T. Łuczak. Turán's extremal problem in random graphs: forbidding odd cycles. Combinatorica, 16(1):107-122, 1996.

[15] Y. Kohayakawa. Szemerédi's regularity lemma for sparse graphs. In Foundations of computational mathematics (Rio de Janeiro, 1997), pages 216-230. Springer, Berlin, 1997.

[16] Y. Kohayakawa and B. Kreuter. Threshold functions for asymmetric Ramsey properties involving cycles. Random Structures Algorithms, 11(3):245-276, 1997.

[17] Y. Kohayakawa, B. Kreuter, and A. Steger. An extremal problem for random graphs and the number of graphs with large even-girth. Combinatorica, 18(1):101-120, 1998.

[18] Y. Kohayakawa, T. Łuczak, and V. Rödl. On $K^{4}$-free subgraphs of random graphs. Combinatorica, 17(2):173-213, 1997.

[19] Y. Kohayakawa and V. Rödl. Szemerédi's regularity lemma and quasirandomness. In Recent advances in algorithms and combinatorics, volume 11 of CMS Books Math./Ouvrages Math. SMC, pages 289-351. Springer, New York, 2003.

[20] Y. Kohayakawa, V. Rödl, and M. Schacht. The Turán theorem for random graphs. Combin. Probab. Comput., 13(1):61-91, 2004.

[21] M. Marciniszyn, J. Skokan, R. Spöhel, and A. Steger. Threshold functions for asymmetric Ramsey properties involving cliques. In Approximation, randomization and combinatorial optimization, volume 4110 of Lecture Notes in Comput. Sci., pages 462-474. Springer, Berlin, 2006. 
[22] L. Pósa. Hamiltonian circuits in random graphs. Discrete Math., 14(4):359-364, 1976.

[23] M. Simonovits. Paul Erdős' influence on extremal graph theory. In The mathematics of Paul Erdös, II, volume 14 of Algorithms Combin., pages 148-192. Springer, Berlin, 1997.

[24] B. Sudakov and V. H. Vu. Resilience of graphs. Random Structures \&5 Algorithms. To appear.

[25] T. Szabó and V. H. Vu. Turán's theorem in sparse random graphs. Random Structures \& Algorithms, 23(3):225-234, 2003.

[26] D. R. Woodall. Sufficient conditions for circuits in graphs. Proc. London Math. Soc. (3), 24:739-755, 1972. 